\title{
MOTIVATION OF PARTICIPANTS IN CROWDSOURCING PLATFORMS USING INTELLIGENT AGENTS
}

\author{
Vijayalakshmi Anand 1), Chittaranjan Hota 2) \\ 1) Birla Institute of Technology \& Science - Pilani \\ Pune Off-Campus Centre \\ Near Bandal Estate, PUNE - 411 021, Maharashtra, India. \\ vijayalakshmi_anand@pilani.bits-pilani.ac.in \\ ${ }^{2)}$ Birla Institute of Technology \& Science, Pilani \\ Department of Computer Sc. and Information Systems \\ Hyderabad Campus, HYDERABAD - 500 078, Telangana, India. \\ hota@hyderabad.bits-pilani.ac.in
}

Paper history:

Received 1 May 2019

Received in revised form 22 November 2019

Accepted 17 February 2020

Available online 31 March

Keywords:

Crowdsourcing;

Social media;

Motivation;

Machine learning;

Q learning algorithm.

\begin{abstract}
Crowdsourcing is a model where individuals or organizations receive services from a large group of Internet users including ideas, finances, completing a complex task, etc. Several crowdsourcing websites have failed due to lack of user participation; hence, the success of crowdsourcing platforms is manifested by the mass of user participation. However, an issue of motivating users to participate in crowdsourcing platform stays challenging. We have proposed a new approach, i.e., reinforcement learning-based gamification method to motivate users. Gamification has been a practical approach to engaging users in many fields, but still, it needs an improvement in the Crowdsourcing platform. In this paper, the gamification approach is strengthened by a reinforcement learning algorithm. We have created an intelligent agent using the Reinforcement learning algorithm (Q-learning). This agent suggests an optimal action plan that yields maximum reward points to the users for their active participation in the Crowdsourcing application. Also, its performance is compared with the SARSA algorithm (On- policy learning), which is another Reinforcement learning algorithm.
\end{abstract}

Copyright $(\odot$ Research Institute for Intelligent Computer Systems, 2020. All rights reserved.

\section{INTRODUCTION}

Recently, crowdsourcing has emerged as a cutting-edge problem-solving platform for the business where many people are involved in solving the complex problems that machines would not solve. Crowdsourcing is the process of resolving or solving a complex problem by many people (and mostly online). The advantage of using crowdsourcing in business is that millions of people with diversified knowledge can share their inputs or expertise to solve a complex problem, and some of these solutions provided are found better than an expert's answer in some cases. Crowdsourcing platform is active with a different focus in many fields such as Amazon Mechanical Turk, Wikipedia, Threadless.com, IStockPhoto, and Galaxy zoo, etc.
Crowdsourcing works excellent on the participation of people's count to solve the problem. However, many crowdsourcing projects are distorted due to a lack of people's involvement [1]. Hence, motivating people to participate in a crowdsourcing platform is the primary challenge in using it. The basic idea of motivation is to attract people to do any action or task. There are two types of motivations used in crowdsourcing platforms, i.e., intrinsic motivation and extrinsic motivation.

In Intrinsic motivation, an individual gets motivated internally, i.e., just by doing the task without expecting any external reward. In the case of extrinsic motivation, an individual anticipates something externally to do the work like reputation, money, etc. [2]. Jakob Nielsen conducted a study on online communities and concluded that only a small 
fraction of users are involved in contributing to such platforms. The majority of the users do not show a lot of interest [3]. It becomes beneficial that the motivators or incentives to participation need to be deeply understood [4].

Many researchers have studied the consequences of intrinsic motivation and extrinsic motivation in the crowdsourcing platform [5], [6], [7], [8] and found that money plays a significant role in attracting individuals at the beginning. Some researchers have analyzed the famous crowdsourcing platform Amazon Mechanical Turk and concluded that the quality of work submitted by participants has not increased because of high monetary reward, and intrinsic motivation helps to improve the quality of work [2]. As there is no standard pricing structure, it rather spoils the relationship between the requester and the worker. Further, the high budget tasks are more lucrative to many workers than the low budget tasks [9]. It was observed that typically, financial rewards degrade the performance of a worker when put next to no reward system [10]. Etzioni [11] concludes that the monetary reward is not good in every situation and also spoils the intrinsic motivation of the workers. Hence, in this research, we have proposed gamification techniques to motivate the people in the crowdsourcing platform.

Gamification technique is a way to attract people and improve their participation. The use of gamification may keep away from the circumstance where a publicly supporting task may fail due to fewer people participating. Henceforth, how to apply gamification properly to build support and commitment has turned into a recent research topic [12].

Generally, the use of game elements in nongame context to encourage desired behaviors of participants is called gamification. In a learning system, game elements such as points, levels, badges, etc. are generally used as incentives. Hence the primary aspect of gamification is rewarding, which provides extrinsic motivation to the user. Moreover, extrinsic motivation is not necessarily considered in financial terms, and it can be nonfinancial also. The problem with extrinsic motivation is that because most of the platforms provide less amount of money than remuneration after completing the work. Hence, always people who participate in crowdsourcing work will doubt whether their work will be accepted or rejected, saying that it is an unsatisfactory work. And some crowdsourcing sites even do not provide anything, so how to motivate people to participate in crowdsourcing platform is a primary challenge in the crowdsourcing platform. We have attempted to address this problem in this work.

The rest of the paper is made up of the following sections. Section 2 consists of related work which discusses some of the recent works in this area carried out by other researchers. Section 3 provides the background required to help understand the next section. Section 4 describes the proposed system. In section 5, we describe the experimentation details and the results obtained. Section 6 concludes our work.

\section{RELATED WORK}

Gamification has been used by scholars and practitioners in different contexts and different fields such as marketing, social networking, and learning.

J. Goncalves used crowdsourcing and gamification to create the keyword dictionary to describe locations on public displays [4]. In [13], the image-labeling game called, Wordsmith was designed for the experimental game conditions. The gamification techniques are used to attract and retain many reliable workers for crowdsourcing tasks like relevance assessments and clustering [14]. Another researcher explored the possibility of engaging a secondary group of millennials, who are notorious technology enthusiasts, with a gamified citizen science application named as Bio tracker, which is used to collect the plant phenology data [15].

Since the number of employees who typically reply to survey requests is generally low, hence, Smith and Kilty used game elements along with crowdsourcing to encourage the employees to respond to online enterprise meetings about software quality survey requests [16]. These days, gamification is also being utilized in requirement engineering in many software companies [17].

In [18], a large amount of data is collected from a heterogeneous population for the study of touchscreen operation in natural environments using the gamification technique. A gamified crowdsourcing system named as Quizz is developed, which is used to assess the knowledge of users and gain new insights from them. Quizz works by requesting that the clients' complete short tests on specific subjects when the client addresses the test questions, Quizz evaluates the client's ability [19].

B. Morschheuser et al. explored how unique gamification techniques build crowd's inspiration and participation in crowdsourcing tasks. His empirical study proved that the gamification had been a practical approach for expanding the people's engagement in crowdsourcing [20]. Another researcher presented G.A.M.E., which is a structure to manage the plan of gamification in crowdsourcing 
based frameworks. The structure gave an adaptable bit-by-bit rule that consolidated information from software engineering, shared courses of action, game plan, and communication structure [21]. Microsoft created a code hunt. It is a web-based gaming platform for the participation of people to do crowdsourcing tasks [22].

The above study indicates that gamification is used in many applications in various domains, but still needs improvement. In this paper, we have used the Reinforcement learning algorithm based on intelligent agents to fortify the gamification technique. The reinforcement learning algorithm has been already used in crowdsourcing for various purposes, like in task assignment and incentive design [23], [24], [25], [26]. Some researchers and physiologists tried to understand the intrinsic motivation based on behavioral theory and tried to create an intrinsic motivation model. In [27], the authors explained that intrinsic motivation is used as an internal reward mechanism that goes well with a simple reinforcement learning algorithm in cognitive computing. Both the internal reward and Reinforcement learning algorithm can be used in an autonomous learning system. The simplest Reinforcement learning is based on the idea of Thorndike's law of effect [28]. If any activity is trailed by an improvement or fulfillment in the state of activities, at that point, the inclination to deliver that activity is fortified. Redgrave and Gurney explained the relationship between intrinsic motivations and Dopamine [29]. Dopamine is a neuron that activates the brain to generate the learning signal. Like this, many researchers have worked on the problem of implementing intrinsic motivation in terms of reinforcement learning algorithms [30], [31], [32], [33].

It is clear from the above study that there is a strong relationship between intrinsic motivation and Reinforcement learning. The reinforcement learning gives predictions based on intrinsic motivation, which maximizes the interest by providing data with reduced subjective complexity and is based on neuroscientific theory on action discovery and learning. Intrinsic motivation learning signals will be released by the brain during the prediction of future states based on current states. This concept is well suited to the motivation of user participation in crowdsourcing activities.

Hence, we have created an intelligent agent using Reinforcement learning and gamification technique. The reinforcement learning algorithm gives the motivation signal during the prediction of future action, and gamification technique gives virtual rewards to motivate the engagement of the user in crowdsourcing activities externally.

\section{CONCEPTUAL FOUNDATION}

In this section, the definition of the gamification technique and reinforcement learning algorithm is explained briefly.

\subsection{GAMIFICATION TECHNIQUE}

In a non-gaming context, the utilization of game components and game mechanics is called the gamification technique [34]. In Werbach and Hunter [35] pyramid, game elements are organized in three classes: dynamics, mechanics, and components. The elements in dynamics group are: progression, emotions, constraints, and relationships. The element in mechanics drives user engagement with content such as feedback, challenge, cooperation, and competition. The tools used to motivate users in the environment of interest are components including achievement, badge, combat, leaderboard, and level. In our research, points and golden reward are used as a game element to motivate the user to participate in the crowdsourcing platform.

\subsection{REINFORCEMENT LEARNING ALGORITHM}

Markov decision process is a simple and progressive decision-making process under uncertainty circumstances [36]. The reinforcement learning frame work is similar to Markov decision process. Like the Markov decision process, the reinforcement learning algorithm consists of states, actions, and rewards in an uncertain environment. The responses received from the environment are represented by states, for example, contributions from the agents. The activities involved by an agent in the environment is represented by the actions. Responses by the actions create reward or punishment [37]. The learning happens through positive feedback or negative feedback in the reinforcement learning algorithm. The reinforcement learning algorithm allows agents to take decision on the perfect actions naturally inside a particular environment, so as to increase its reward. The reinforcement signal, the simple reward, is required for the agent to learn the actions. One of the important reinforcement learning algorithms is the Q-learning algorithm. We have used the Q-learning algorithm to create the intelligent agent.

\subsection{Q FUNCTION}

As Watkins [38] mentioned, we can define Reward and policy as below.

Reward: In Markov Decision Process (MDP), the movement of agent from one state to another state depends on the current state $S_{t}$ and reward $R_{t}$. Since 
the environment is stochastic, the total reward $R_{t}$ received from the current time step $t$ to the end of the task can be defined in terms of discounted reward.

$$
\begin{aligned}
\mathrm{R}_{\mathrm{t}} & =\mathrm{R}_{\mathrm{t}+1}+\gamma \mathrm{R}_{\mathrm{t}+2}+\gamma^{2} \mathrm{R}_{\mathrm{t}+3} \\
& =\sum_{\mathrm{k}=0}^{\infty} \gamma^{\mathrm{k}} \mathrm{R}_{\mathrm{t}+\mathrm{k}+1},
\end{aligned}
$$

where $0<\Upsilon<1$ is the discount factor.

Policy: Policy $\pi$ is defined as the probability of selecting the best action for the given state. It is mathematically described as:

$$
\pi(\mathrm{a} \mid \mathrm{s})=\mathrm{P}\left[\mathrm{A}_{\mathrm{t}}=\mathrm{a} \mid \mathrm{S}_{\mathrm{t}}=\mathrm{s}\right]
$$

There are two types of value functions, i.e., state value function and action value function. State value function $V_{\pi}(s)$ is defined as long term reward of $s$ after following policy $\pi$. It is described as:

$$
\mathrm{V}_{\pi}(\mathrm{s})=\mathrm{E}\left[\mathrm{R}_{\mathrm{t}} \mid \mathrm{S}_{\mathrm{t}}=\mathrm{s}\right],
$$

Action value function $\mathrm{q}_{\pi}(\mathrm{s}, \mathrm{a})$ is defined as the expected reward for the given action (a) at a particular state(s) by following the policy $\pi$.

$$
\mathrm{q}_{\pi}(\mathrm{s}, \mathrm{a})=\mathrm{E}_{\pi}\left[\mathrm{R}_{\mathrm{t}} \mid \mathrm{S}_{\mathrm{t}}=\mathrm{s}, \mathrm{A}_{\mathrm{t}}=\mathrm{a}\right] .
$$

Optimal function for the Bellman equations is as given in equations 7 and 8.

$$
\begin{gathered}
\mathrm{V}_{\pi}(\mathrm{s})=\sum_{\mathrm{a} \in \mathrm{A}} \pi(\mathrm{a} \mid \mathrm{s}) \mathrm{q}_{\pi}(\mathrm{s}, \mathrm{a}) \\
\mathrm{q}_{\pi}(\mathrm{s}, \mathrm{a})=\mathrm{R}_{\mathrm{s}}^{\mathrm{a}}+\gamma \sum_{\mathrm{s}^{\prime} \in \mathrm{S}} \mathrm{P}_{\mathrm{s} \mathrm{s}^{\prime}}^{\mathrm{a}} \mathrm{v}_{\pi}\left(\mathrm{s}^{\prime}\right)
\end{gathered}
$$

Optimal function for the Bellman equations is

$$
\begin{aligned}
& \mathrm{v} *(\mathrm{~s})=\max _{\mathrm{a}}\left(\mathrm{R}_{\mathrm{s}}^{\mathrm{a}}+\gamma \sum_{\mathrm{s}^{\prime} \in \mathrm{S}} \mathrm{P}_{\mathrm{ss}^{\prime}}^{\mathrm{a}} \mathrm{v}_{\mathrm{s}}\left(\mathrm{s}^{\prime}\right)\right) \\
& \mathrm{q}^{*}(\mathrm{~s}, \mathrm{a})=\mathrm{R}_{\mathrm{s}}^{\mathrm{a}}+\gamma \sum_{\mathrm{s}^{\prime} \in \mathrm{S}} \mathrm{P}_{\mathrm{ss}^{\prime}}^{\mathrm{a}} \max _{\mathrm{a}^{\prime}} \mathrm{q}\left(\mathrm{s}^{\prime} \mathrm{a}^{\prime}\right)
\end{aligned}
$$

We can derive the $\mathrm{Q}$ value as a weighted average of old $Q$ value and new $Q$ value at time step t.

As shown in Fig. 2, the proposed system consists of states (S), actions (A), and reward (R). Like an agent works in an environment in the reinforcement learning, a faculty in the faculty network acts as an agent. Faculty learns from the faculty network by doing a set of actions and collecting positive or negative reward at a particular state. Actions performed by faculties in the faculty network

$$
\begin{aligned}
Q\left(s_{t}, a_{t}\right) \rightarrow & Q\left(s_{t}, a_{t}\right)+\propto\left(r_{t+1}+\right. \\
& \gamma \max _{a}\left(Q\left(s_{t+1}, a\right)-Q\left(s_{t}, a_{t}\right)\right)
\end{aligned}
$$

Where, $Q\left(s_{t+1}, a_{t+1}\right), Q\left(s_{t}, a_{t}\right)$ are new and old Q-values for the action (a) at state (s). Here, $\alpha$ and $\gamma$ are the learning rates, and discount factor and the value remains in the range $(0,1)$ interval, where $r$ is the reward for carrying out the action (a) in the state(s).

\section{PROPOSED SYSTEM}

We have used the academic domain as a case study to show the performance of the proposed method. It is very well fit with our research scenario as the university/colleges consist of many people like students, professors, and administrative staff, etc. Normally the problems in an academic domain like colleges and universities are solved by arranging experts or consultants or collecting feedback from people. In our research, we show that crowdsourcing is a more suitable technique for solving problems in the academic domain; hence, we have created a faculty network, a social media application, for professors to share their educational and research experience. This faculty network acts as a platform for collaborative tasks. Faculty network holds various modules like forums, articles, blogs, and a task manager. The task manager module, as shown in Fig. 1 of the faculty network, is used for crowdsourcing activities like requester can post the task, and workers can apply for solving the task.

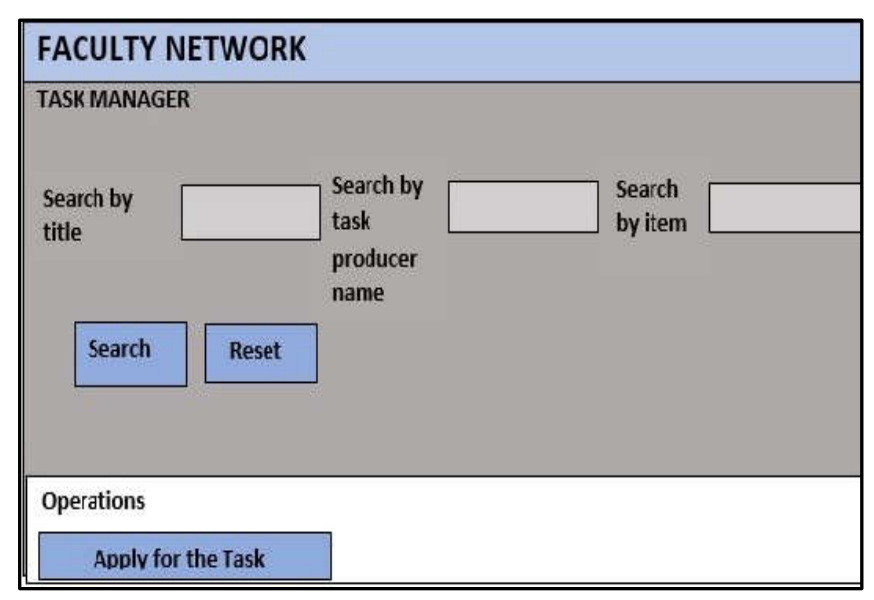

Figure 1 - Screenshot of Task manager

include the following ones: post the task, post the question and post the answer, post the blog and post the article as the faculty network is created to share the academic and research experience of faculties. If faculty likes other faculty's posts (questions, answers, blogs, and articles), he or she can vote it up, or if he or she doesn't like it, they can vote it down. All states and activities will be attempted 
persistently. Lastly, the best activity and reward will be selected.

The Q-learning algorithm works as follows: there is a Q table that will be updated by the state, corresponding actions, and rewards for each iteration. At every time period $t$, the agent selects its state $s \in \mathrm{S}$ from the set of states and picks an action a $\epsilon$ A from the set of actions and a reward $R$ dependent on the up vote and down vote in a particular state. Activities are chosen based on a policy $\pi: \mathrm{S} \rightarrow \mathrm{A}$ that is changed after some time as the agent attempts different activities and gathers proportionate rewards after numerous attempts, the total reward will be increased using the below reward function: $\mathrm{S} \times \mathrm{A} \rightarrow \mathrm{R}$.

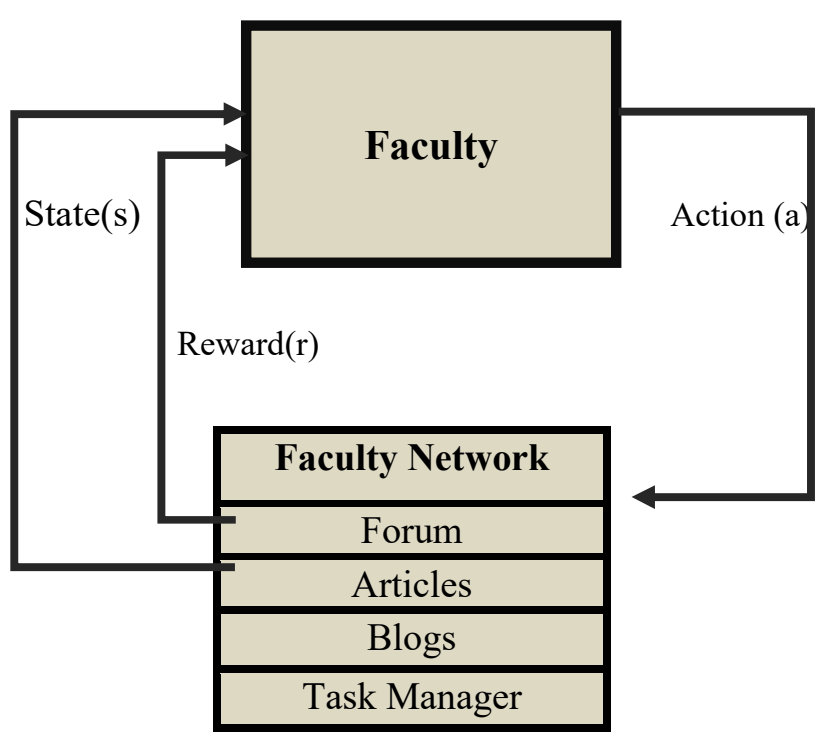

Figure 2 - Proposed System

Consider the states $\mathrm{S}=\left\{\mathrm{s}_{0}=0-50, \mathrm{~s}_{1}=51-100\right.$, $\left.\mathrm{s}_{2}=101-150 \ldots \ldots \mathrm{s}_{\mathrm{k}}=951-1000\right\}$ and actions $\mathrm{A}=$ ppost the question $\mathrm{Q}$, post the answer An, post the blog content B or Article Ar and post the task T $\}$ and also assume that initially the reward $\mathrm{R}=1$ and then based on up votes (Uv) and Downvotes (Dv) the reward $R$ is calculated by the formula specified in Algo. 1 .

\subsection{Q-LEARNING ALGORITHM}

In the Q-learning algorithm, the agent walks around from one state to another state until it touches the goal state and thus converges. The goal state is the last (1000 points), as mentioned in the above paragraph. The faculty receives the golden reward at the goal state.

The state $\left(\mathrm{S}_{t}\right)$ and action $\left(\mathrm{A}_{\mathrm{t}}\right)$ pairs are updated in Q-table at each time step until it converges.

Now, the Q-table shows the optimal action for each state. The steps used in the Q-learning algorithm are shown in Algorithm 1. Initially, the values of reward $(\mathrm{R})$ is 0 . Then, the reward is observed for the actions performed at the particular state $(\mathrm{S})$. The action that has maximum $(\mathrm{Q})$ value is selected.

\section{Algorithm 1:Q-Learning algorithm}

$$
\begin{gathered}
\text { Input: } \text { States } S=\{\mathrm{s} 0, \mathrm{~s} 1, \mathrm{~s} 2 \ldots \ldots \mathrm{s} 19\} \\
\text { Actions } \mathrm{A}=\mathrm{Ar}, \mathrm{B}, \mathrm{T}, \mathrm{Q}, \mathrm{An} \\
\mathrm{Q}(\mathrm{s}, \mathrm{a})=0 \\
\mathrm{r}-0.9, \alpha \leftarrow 0.1
\end{gathered}
$$

Output:Q(s,a) Qvalue at each iteration

1 :for each iteration do

2: $\quad$ select random state $\mathrm{s}_{\mathrm{t}} \leftarrow \mathrm{S}$ (Egreedy Policy)

3. for each step in the iteration do

4: $\quad$ choose action $\mathrm{a}_{\mathrm{t}} \leftarrow \mathrm{s}_{\mathrm{t}}$ (Using derived policy)

5: $\quad$ observe reward $\mathrm{r}_{\mathrm{t}+1} \leftarrow \mathrm{r}+\mathrm{Uv}+\mathrm{Dv}$

6: $\quad$ observe new state st-st +1

7: $\quad$ update Q value based on Eq. (9)

8: $\quad \mathrm{s} \leftarrow \mathrm{S}_{\mathrm{t}+1}$

9: $\quad$ end for

10: end for

\section{EXPERIMENTAL SETUP AND RESULTS}

As mentioned in Section 3, the faculty network has been implemented to test our motivation algorithm. Around 650 faculties were requested to register in the faculty network from our university. It is a social media-based crowdsourcing platform. In this network, faculties can share their academic and research experiences and can perform crowdsourcing activities too. The advantages of using social media crowdsourcing platform is to improve intrinsic motivation like peer review, connections with the experts, etc.

We have formed an intelligent agent using Q learning. This intelligent agent, which is used to motivate the faculties to participate in crowdsourcing activities, is attached to our Faculty network. The intelligent agent works as follows: It consists of a $\mathrm{Q}$ table which does not contain any value. $Q$ table is updated on every iteration until it converges. Here, learning is done by the actions performed by the faculty at a particular state. Its value is evaluated in terms of the Up vote or Down vote it receives. Up vote receives +1 point, and Down vote gains -1 point. By trying all the actions (A) in all states (S) continually, the model learns the best actions for each state by long term discounted 
reward. Table1 shows the simulation parameter used to create intelligent agent.

Table1. Simulation Parameters.

\begin{tabular}{|l|l|}
\hline Parameter & \multicolumn{1}{|c|}{ Values } \\
\hline Up votes & 1 \\
\hline Down votes & -1 \\
\hline$\Upsilon$ & 0.9 \\
\hline$\alpha$ & 0.1 \\
\hline Maximum number of iteration & 20000 \\
\hline
\end{tabular}

Here epsilon-greedy policy is used to select the actions which work based on exploration and exploitation. We would like it to take random action to discover further actions as an agent starts learning. Once the agent learns, the Q-function converges to Q-values. Now our agents take advantage of the highest Q-value i.e., takes greedy actions. During the exploration phase, it tries all the possible states and actions, and after completing enough number of iterations, it learns the correct action for a particular state. Agent selects random action for probability $\varepsilon$ and greedy action for probability $(1-\varepsilon)[39]$. It is necessary. Otherwise, it stuck in local optimum, and will never find an improved policy.

The performance of the Q-learning algorithm is presented in Fig. 3 and Fig. 4. From the figure, one can realize that our algorithm converges after some iterations and provides the optimal action for each state. Also, we have shown the sample policy in Table 2. This model informs the faculty on which actions give maximum reward and reach the goal state from any state easily. The goal is to achieve1000 points. Once the faculty receives 1000 reward points, he or she is eligible to get a golden reward and some privileges. This strengthens the gamification technique.

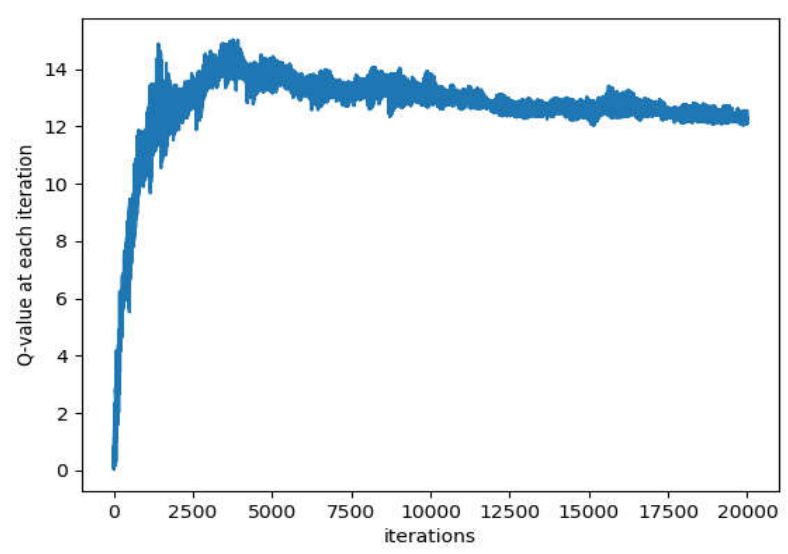

Figure 3 - Convergence of Q-learning algorithm

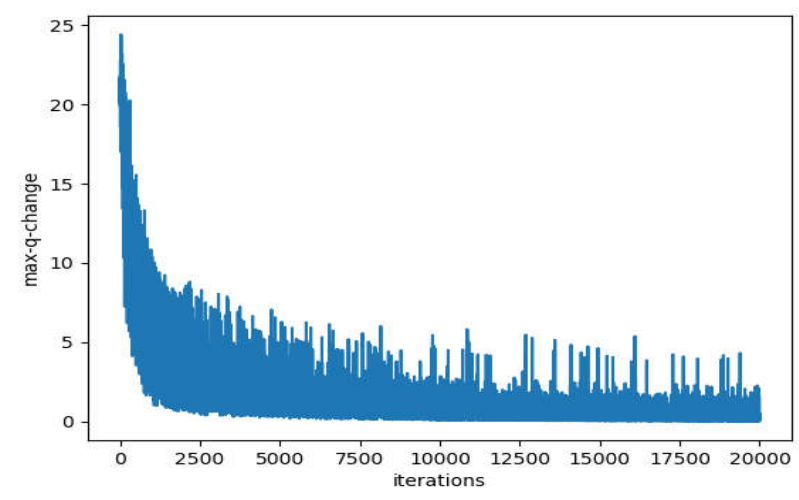

Figure 4- $Q$ value vs Iterations

In our experiment, we have also matched the performance of the Q-learning algorithm with another famous reinforcement learning algorithm [40]. Fig. 5 shows the convergence of an intelligent agent using the SARSA algorithm. The Q-learning is an offline policy, and the SARSA algorithm is an online policy algorithm. The main difference between these two algorithms is how the Q-value is updated. In SARSA, learning is done based on the action performed by the current policy, but in Qlearning, the action with maximum reward is chosen.

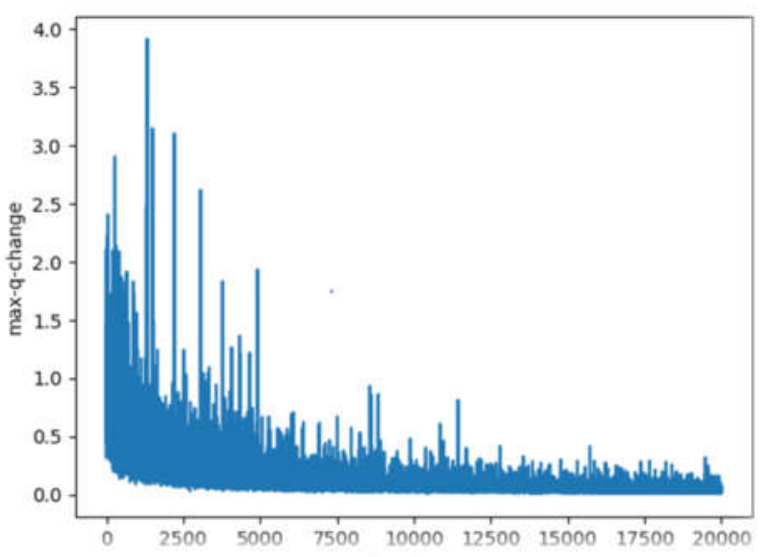

Figure 5 - Convergence of Sarsa algorithm

$$
\begin{aligned}
& Q\left(s_{t}, a_{t}\right) \rightarrow Q\left(s_{t}, a_{t}\right)+\propto\left(r_{t+1}\right. \\
& +\gamma\left(Q\left(s_{t+1}, a_{t+1}\right)-Q\left(s_{t}, a_{t}\right)\right)
\end{aligned}
$$

We understand that both algorithms converge after some iterations. The Sarsa algorithm converges faster than the Q-learning algorithm. Though it converges fast, it does not perform well, i.e., cannot find optimal path planning.

Although both converge at optimal policy, Qlearning is suitable for low cost and the fast iterating environment as it learns optimal policy directly. 


\section{Algorithm 2:SARSA algorithm}

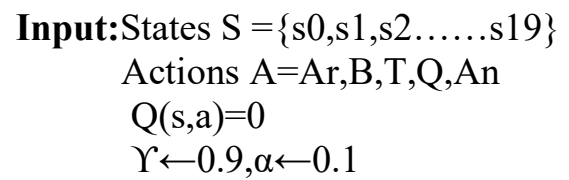

Output:Q(s,a) Qvalue at each iteration

1 :for each iteration do

2: select random state $\mathrm{s}_{\mathrm{t}} \leftarrow \mathrm{S}$

3: choose action $\mathrm{a}_{\mathrm{t}} \leftarrow \mathrm{s}_{\mathrm{t}}$

4. for each step in the iteration do

5: $\quad$ choose action $\mathrm{a}_{\mathrm{t}} \leftarrow \mathrm{S}_{\mathrm{t}}$ (Using derived policy)
6: $\quad$ observe reward $\mathrm{r}_{\mathrm{t}+1} \leftarrow \mathrm{r}+\mathrm{Uv}+\mathrm{Dv}$
7: $\quad$ observe new state $\mathrm{s}_{\mathrm{t}}-\mathrm{S}_{\mathrm{t}+1}$
8: $\quad$ update Q value based on Eq.( 10)
9: $\quad \mathrm{s} \leftarrow \mathrm{s}_{\mathrm{t}+1}$
10: $\quad \mathrm{a} \leftarrow \mathrm{a}_{\mathrm{t}+1}$
11: end for
12: end for

So, we have used the Q-learning algorithm in the faculty network for motivating the faculties in participating in the crowdsourcing activities.

We have attached the intelligent agent to the faculty network and observed the policy obtained at each state. When a faculty login to the system, it suggests some action to do, which yields maximum reward based on this table. For example, if the faculty is in state S2, the system will indicate the faculty to do action "post an answer."

Further, we have checked the response rate of the Q- learning based intelligent agent on faculty network and observed that the number of responses for our approach increased than the cash prize which is shown in Fig. 6.

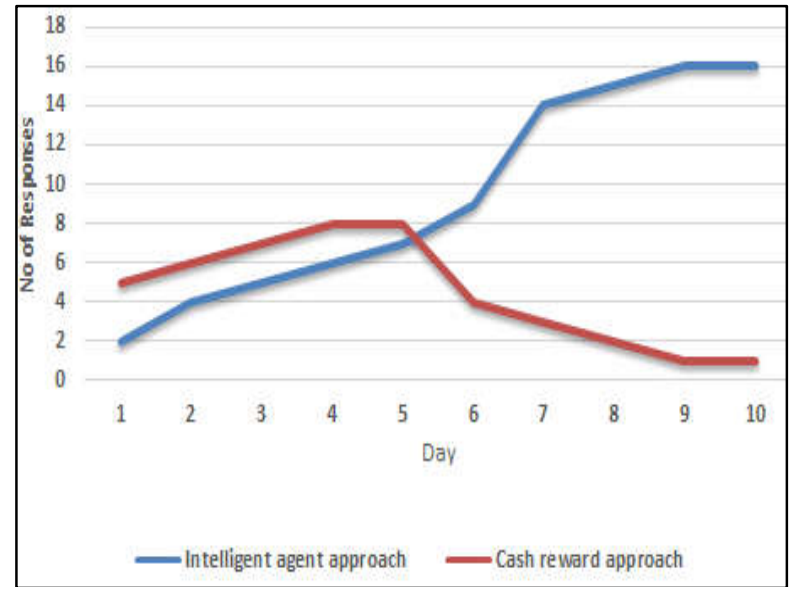

Figure 6-Response rate of $Q$ learning algorithm
Table2. Sample policy for Qlearning algorithm

\begin{tabular}{|c|c|}
\hline State & Actions \\
\hline S0 & Post an article(Ar) \\
\hline S1 & Post a task(T) \\
\hline S2 & Post an answer(An) \\
\hline S3 & Post an answer(An) \\
\hline S4 & Post an article(Ar) \\
\hline S5 & Post a task(T) \\
\hline S6 & Post an blog(B) \\
\hline S7 & Post an article(Ar) \\
\hline S8 & Post a Question(Q) \\
\hline S9 & Post an blog(B) \\
\hline S10 & Post an answer(An) \\
\hline S11 & Post a task(T) \\
\hline S12 & Post an article(Ar) \\
\hline S13 & Post a task(T) \\
\hline S14 & Post an answer(An) \\
\hline S15 & Post a task(T) \\
\hline S16 & Post an article(Ar) \\
\hline S17 & Post a task(T) \\
\hline S18 & Post an article(Ar) \\
\hline S19 & Post an answer(An) \\
\hline
\end{tabular}

The screenshot of the reward details of one faculty on Faculty network is shown in the Fig. 7.

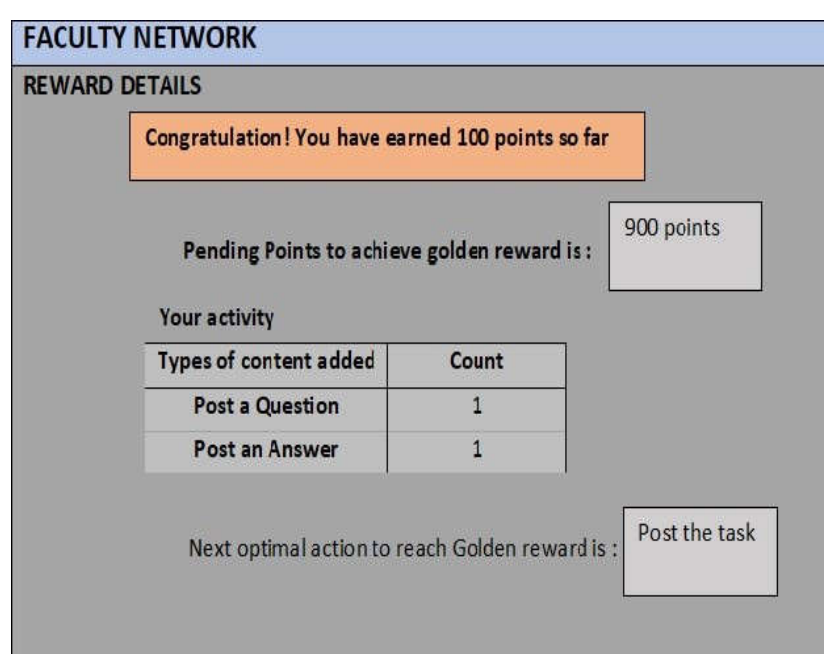

Figure 7 - Reward details of Faculty Network

\section{CONCLUSIONS AND FUTURE WORK}

The proposed technique for the motivation of user participation in the crowdsourcing platform uses both the gamification element and Q learning algorithm. This is more effective than simply using gamification elements as this framework boosts the intrinsic motivation of the people to engage in crowdsourcing activities.

Further, Gamification with Reinforcement learning can make a customized understanding for users in crowdsourcing platform that will not just 
keep them progressively connected yet offer the open door for persistent learning and to successfully change their conduct when required. The limitation of our algorithm is, here, just for simplicity, we have considered the goal state for an agent is to reach 1000 points. At this goal state, the user will receive a golden reward. But in the future, we would like to improve our system by exploring more on rewards and privileges once the faculties received a golden reward. The problem with $\mathrm{Q}$ learning is how to balance the exploration and exploitation dilemma, increase the convergence rate, and abstain from converging to a local optimum.

\section{ACKNOLWEDGEMENT}

We thank our off-campus faculty colleagues who have volunteered to participate in the social media application deployed in the faculty network, giving us an opportunity to gather real world datasets in an academic environment.

\section{REFERENCES}

[1] Z. Zeng, J. Tang, T. Wang, "Motivation mechanism of gamification in crowdsourcing projects," International Journal of Crowd Science, vol. 1, issue 1, pp. 71-82, 2017.

[2] N. Kaufmann, T. Schulze, D. Veit, "More than fun and money. Worker motivation in crowdsourcing - A study on mechanical Turk," Proceedings of the Americas Conference on Information Systems (AMCIS), Detroit, Michigan, 4-8 August 2011, pp. 1-11.

[3] J. Nielsen, "The 90-9-1 rule for participation in social media and online communities," in: N/Ng Nilsen Normal Group Articles, Oct 8, 2006.

[4] J. Goncalves, S. Hosio, D. Ferreira, V. Kostakos, "Game of words, tagging places through crowdsourcing on public displays," Proceedings of Designing Interactive systems (DIS), Vancouver, BC, Canada, 21-25 June 2014, pp. 705-714.

[5] D. Difallah, E. Filatova, P. Ipeirotis, "Demographics and dynamics of mechanical Turk workers," Proceedings of the Eleventh ACM International Conference on Web Search and Data Mining WSDM'2018, USA, February 5-9, 2018, pp.135-143.

[6] R. M. Ryan, E.L. Deci, "Intrinsic and extrinsic motivations: Classic definition and new directions," Contemporary Educational Psychology, vol. 25, issue 1, pp. 54-67, 2000.

[7] J. Rogstadius, V. Kostakos, A. Kittur, B. Smus, J. Laredo and M. Vukovic, "An assessment of intrinsic and extrinsic motivation on task performance in crowdsourcing markets,"
Proceedings of the Fifth International Conference on Weblogs and Social Media, Barcelona, Catalonia, Spain, July 17-21, 2011, pp. 321-328.

[8] D.C. Brabham, "Crowdsourcing as a model for problem solving: An introduction and cases Convergence," The International Journal of Research into New Media Technologies, vol. 14, issue 1, pp. 75-90, 2008.

[9] Yu Zhang, Michaela Vander Schaar, "Reputation-based incentive protocols in crowdsourcing applications," Proceedings of the IEEE INFOCOM, 2012, pp. 2140-2148.

[10] B. A. Hennessey, "The social psychology of creativity," Scandinavian Journal of Educational Research, vol. 47, issue 3, pp. 253-271, 2010.

[11] A. Etzoni, Modern Organizations, Eaglewood Cliffs, NJ-Prentice Hall, 1964, 120 p.

[12] R. S. Alsawaier, "The effect of gamification on motivation and engagement," International Journal of Information and Learning Technology, vol. 35, issue1, pp. 56-79, 2018.

[13] O. Feyisetan, E. Simperl, M.V. Kleek, N. Shadbolt, "Improving paid micro tasks through gamification and adaptive furtherance incentives," Proceedings of the 24th International Conference on World Wide Web, Florence, Italy, May18-22, 2015, pp. 333-343.

[14] C. Fickhoff, C.G. Harris, A.P de Vries and S. Padmini, "Quality through flow and immersion gamifying crowdsourced relevance assessments," Proceedings of the 35th international ACM SIGIR Conference on Research and Development in Information Retrieval, Portland, Oregon, 2012, pp. 871-880.

[15] A. Bowser, D.L. Hansen, Y. He, C. Boston, M. Reid, L. Gunnell, J. Preece, "Using gamification to inspire new citizen science volunteers," Proceedings of the International Conference on Gamified Design, Research and Applications, Toronto, Ontario, Canada, October 2-4, 2013, pp. 18-25.

[16] R. Smith, L.A. Kilty, "Crowdsourcing and gamification of enterprise meeting software quality," Proceedings of the 7th International Conference on Utility and Cloud Computing (UCC), London, 2014, pp. 611-613.

[17] R. Snijders, F. Dalpiaz, M. Hosseini, A. Shahri, R. Ali, "Crowd-centric requirements engineering," Proceedings of the IEEE/ACM 7th International Conference on Utility and Cloud Computing, London, 8-11 Dec 2014, pp. 614-615.

[18] S. Malatini, L.C. Klopfenstein and A. Bogliolo, "Gamification for crowdsourced data collection in mobile usability field studies," Proceedings 
of the 12th Biannual Conference on Italian, Italy, Sep 18-20, 2017, pp. 1-10.

[19] P. Ipeirotis and E. Gabrilovich, "Quizz: targeted crowdsourcing with a billion (potential) users," Proceedings of the 23rd International Conference on World Wide Web $(W W W)$, Seoul, Korea, 2014, pp. 143-154.

[20] B. Morschheuser, J. Hamari, J. Koivisto, "Gamification in crowdsourcing: a review, system sciences (HICSS)," Proceedings of the 49th Hawaii International Conference, Kauai, Hawaii, USA, 2016, pp. 4375-4384.

[21] J. Brito, V. Vieira and A. Duran, "Towards a framework for gamification design on crowdsourcing systems: The G.A.M.E. approach," Proceedings of the 12th International Conference on Information Technology - New Generations, Las Vegas, NV, 2015, pp. 445-450.

[22] T.Xie, J. Bishop, R. Nigel Horspool, N. Tillman, J. de Halleux, "Crowd sourcing code and process via code hunt," Proceedings of the Crowdsourcing in Software Engineering, Florence, Italy, May 16-24, 2015, pp.15-16.

[23] Z. Hu, Y. Liang, J. Zhang, Z. Li, and Y. Liu, "Inference aided reinforcement learning for incentive mechanism design in crowdsourcing," Proceedings of the 32nd International Conference on Neural Information Processing System, Canada, Dec 2018, pp. 5512-5522.

[24] L. Cui, X. Zhao, L. Liu, H. Yu, Yu. Miao, "Complex crowdsourcing task allocation strategies employing supervised and reinforcement learning," International Journal of Crowd Science, vol. 1, pp. 146-160, 2017.

[25] R. Qiao, S. Yan and B. Shen, "A reinforcement learning solution to cold-start problem in software crowdsourcing recommendations," Proceedings of the IEEE International Conference on Progress in Informatics and Computing (PIC), Suzhou, China, 2018, pp. 814.

[26] J. Thangarajah, K. Tuyls, C. Jonker, S. Marsella, "Optimal testing for crowd workers," Proceedings of the 15th International Conference on Autonomous Agents and Multi Agent Systems, Singapore, May 2016, pp. 966974.

[27] N. Siddique, P. Dhakan, I, Rano, K. Kasmarik, "A review of the relationship between novelty, intrinsic motivation and reinforcement learning," Journal of Behavioral Robotics, vol. 8, pp. 58-69, 2017.

[28] E. L. Thorndike, "Laws and hypotheses for behavior," in E. L. Thorndike, Animal
Intelligence, Darien, CT: Hafner Publishing Co, 1970, pp. 241-281.

[29] P. Redgrave, K. Gurney, "The short-latency dopamine signal: A role in discovering novel actions," Nature Review, Neuroscience, vol. 7, pp. 967-975, 2006.

[30] P.Y. Oudeyer, F. Kaplan, "What is intrinsic motivation? A typology of computational approaches," Frontiers in Neuro Robotics, vol. 1, issue 6, pp. 1-14, 2007.

[31] M. Schembri, M. Mirolli, G. Baldassarre, "Evolving internal reinforcers for an intrinsically motivated reinforcement-learning robot," Proceedings of the 6th IEEE International Conference on Development and Learning, 2007, pp. 282-287.

[32] J. Schmidhuber, "Formal theory of creativity, fun, and intrinsic motivation (1990-2010)," IEEE Transactions on Autonomous Mental Development, vol. 2, no. 3, pp. 230-247, Sept. 2010.

[33] P.Y. Oudeyer, F. Kaplan, V. V. Hafner, "Intrinsic motivation systems for autonomous mental development," IEEE Trans. Evolutionary Computation, Vol. 11, issue 2, pp. 265-286, 2007.

[34] S. Deterding, D. Dixon, R. Khaled, L. Nacke, "From game design elements to gamefulness: Defining gamification," Proceedings of the 15th International Academic Mind Trek Conference: Envisioning Future Media Environments, Tampere, Finland, Sep. 28-30, 2011, pp. 9-15.

[35] K. Werbach, D. Hunter, For the Win: How Game Thinking Can Revolutionize Your Business, Philadelphia: Persus Distribution Services, 2012.

[36] C.C. White, D.J. White, "Markov decision processes," European Journal of Operational Research, vol. 39, issue 1, pp. 1-16, 1989.

[37] V. Heidrich-Meisner, M. Lauer, C. Igel, M. Riedmiller, "Reinforcement learning in a nutshell," Proceedings of European Symposium on Artificial Neural Networks, Bruges, Belgium, April 25-27, 2007, pp. 277-288.

[38] J.C.H Watkins, "Technical note: Qlearning," Machine Learning, vol. 8, pp. 279-292, 1992.

[39] V. Kuleshov, D. Precup, "Algorithm for the multi armed bandit problem," Journal of Machine Learning Research, vol. 1, pp. 1-48, 2000.

[40] H. Lima, Y. Kuroe, "Swarm reinforcement learning algorithms based on Sarsa method," Proceedings of the SICE Annual Conference, Tokyo, 2008, pp. 2045-2049. 


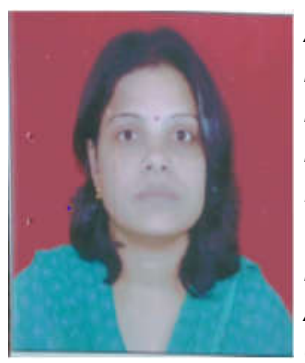

Anand Vijayalakshmi is pursuing PhD in crowdsourcing and machine computation for social media application at BITS Pilani Hyedarbad campus, Hyderabad. She completed her master degree in computer science from Anna University, Chennai and completed her bachelor degree

in Electronics \&communication engineering from Manonmaniam Sundarnar University, Tirunelveli. Currently she is working as an Assistant professor in computer science department at BITS Pilani off campus center, Pune. She has vast teaching experience in Teaching computerscience subjects.

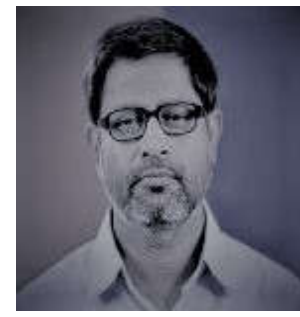

Dr. Chittaranjan Hota, a

Professor of Computer Science and Engineering, completed his Ph.D from BITS, Pilani and has over thirty years of academic and research experience in Indian universities and abroad. His Ph.D work was on Quality of Service (QoS) assurances in IP-Virtual Private Networks, part of which was carried out at University of New South Wales, Sydney, Australia. Prior to $\mathrm{PhD}$, he had earned Bachelors of Engineering in Computer Science, and Masters of Engineering in Computer Science and Engineering. Dr Hota has been in various administartive roles at BITS, Pilani including Associate DEAN, HoD, FIC, and Professor In-Charge over past several years. His research has been funded by MeitY, Govt. of India; TCS; UGC, New Delhi over past several years. He is a Senior Member of IEEE. 